\title{
Spectroscopic Nonadiabatic Observables as a New Tool for Mode Identification in $\beta$ Cep Stars
}

\author{
J. Daszyńska, H. Cugier \\ Astronomical Institute of the Wroctaw University, Poland
}

\begin{abstract}
We present a new method of mode identification based on the line profile variations. We calculate time series of Si III $455.26 \mathrm{~nm}$ for models of $\beta$ Cep stars with a mass of $10 M_{\odot}$. The spectroscopic observables are amplitude ratio and phase difference for various oscillation parameters derived from the line profile changes. Based on the computed values we plot diagrams to search for the best discriminators of modes.

In most spectroscopic diagrams the unstable modes are grouped in well-defined regions with different $m$-values, and a few of them give also separation with respect to $\ell$-values. Moreover, on the basis of almost all of the diagrams we can distinguish the prograde modes from the retrograde ones, and the zonal modes from those with $m \neq 0$.

In this paper we discuss also the influence of the inclination angle, the amplitude of the radius variation and the equatorial velocity on the diagnostic values of the spectroscopic diagrams.
\end{abstract}

\section{Introduction}

It is well known that an essential step for asteroseismology is mode identification, that is, for a given frequency to determine three quantum numbers $n, \ell, m$. One of the most popular techniques of mode identification is the use of photometric nonadiabatic observables, that is photometric amplitudes and phases in selected filters (Watson, 1988; Cugier et al., 1994). Plotting diagrams of type "amplitude ratio versus phase difference" we are able to assign the harmonic degree $\ell$. In the case of $\ell=0$ we can also determine the radial order $n$. However, this method does not give any information about the azimuthal order, $m$.

As first noted by Ledoux (1951) determination of $m$ is possible from an analysis of LPVs. Balona (1986a,b) proposed an algorithmic method of mode identification based on the first few moments of line profiles. Aerts et al. (1992) applied it to the $\beta$ Cep star $\delta$ Ceti.

In this paper we construct diagrams making use of the amplitudes and phases, however both derived from changes of various line profile parameters. For these quantities we adopted the definition spectroscopic nonadiabatic observables by analogy to the photometric nonadiabatic observables.

The paper is organised as follows. In Sect. 2 we describe our model calculations. In Sect. 3, using plots with different observables, we look for the best one to identify the oscillation modes in $\beta$ Cep stars. Conclusions are given in Sect. 4 . 

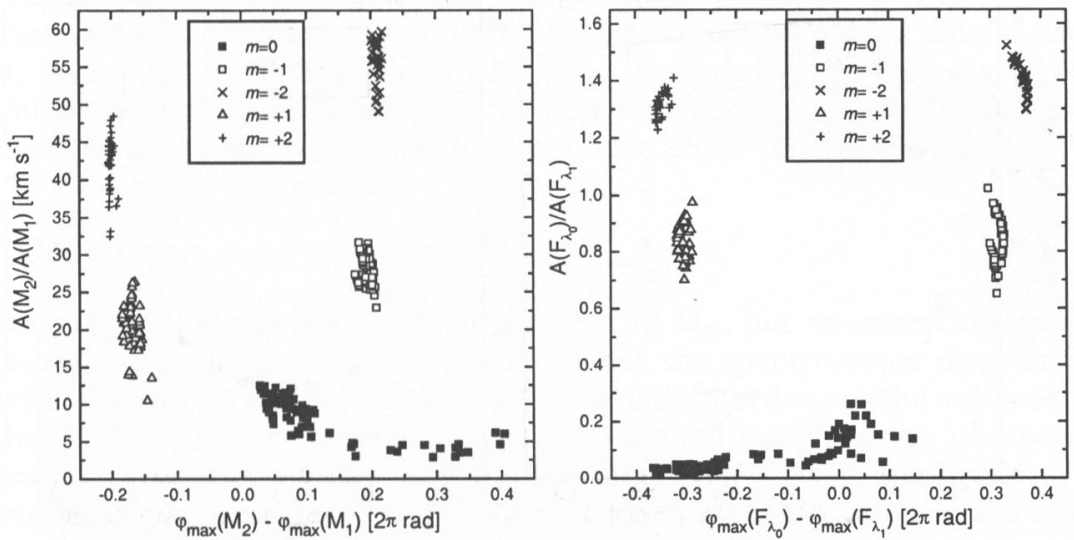

Figure 1. The amplitude ratio versus phase difference for two pairs: the second and first moment (left panel) and the monochromatic fluxes $F\left(\lambda_{0}=455.26 \mathrm{~nm}\right), F\left(\lambda_{1}=\lambda_{0}+0.05\right)$ (right panel) of Si III 455.26. We can see the well-separated domains of modes with different $m$-values.

\section{Model calculations}

We calculated time series of the Si III $455.26 \mathrm{~nm}$ line profiles for $10 M_{\odot}$ Main Sequence models, see Cugier \& Daszyńska (2001) for details. For this purpose we used the linear nonadiabatic results of Dziembowski \& Pamyatnykh (1993) calculated for OPAL opacity tables with $Z=0.02$ and the line-blanketed models of the stellar atmospheres of Kurucz (1996). We took into account unstable modes with low harmonic degree, $\ell=0,1,2$, and with azimuthal order of $m=$ $-\ell, \ldots,+\ell$. We assumed an equatorial velocity $v_{\mathrm{e}}=50 \mathrm{~km} / \mathrm{s}$, an inclination angle $i=60^{\circ}$ and an amplitude of the stellar radius variations $\varepsilon=0.015$. Additionally we assumed the zero-rotation approximation $(\Omega / \omega \approx 0)$, which is appropriate, because we limited our calculations to low rotational velocities, $v_{\mathrm{e}} \leq 50 \mathrm{~km} / \mathrm{s}$. We measured various characteristics of the line profiles as a function of the pulsational phase and fitted a synthetic curve consisting of six sine functions: with the primary period and its first five harmonics. We considered: $F_{\min }$ - residual intensity measured at the deepest point of the normalised line profiles, $E W$ - equivalent width, $F W \cdot H M$ - full width at the half maximum, $F_{\lambda_{0}}$ - line intensity at $\lambda_{0}=455.26 \mathrm{~nm}, F_{\lambda_{1}}$ - line intensity at $\lambda_{1}=\lambda_{0}+0.05 \mathrm{~nm}$, $V_{\min }$ - radial velocity corresponding to $F_{\min }, V_{\mathrm{hm}}$ - radial velocity corresponding to $F W H M$, and $M_{0}, M_{1}, M_{2}$ and $M_{3}$ - zeroth and the first three moments of the line profile.

\section{Mode identification}

We constructed various spectroscopic nonadiabatic diagrams for the primary period in order to search for the best discriminators of oscillation modes in $\beta$ Cep stars. These diagrams appeared to be very useful for the identification of the 


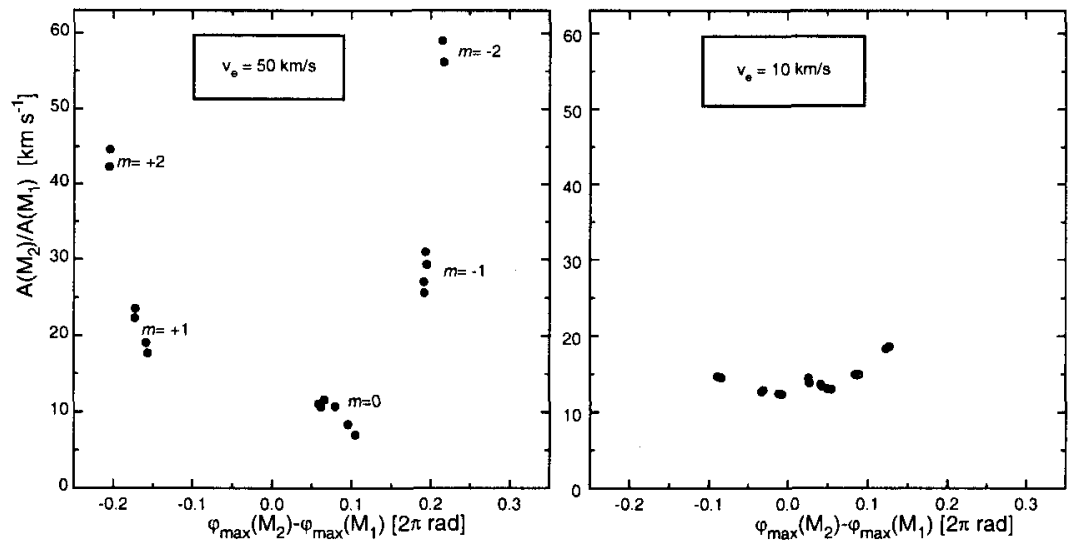

Figure 2. The effect of the equatorial rotation on the diagram $A\left(M_{2}\right) / A\left(M_{1}\right)$ versus $\varphi_{\max }\left(M_{2}\right)-\varphi_{\max }\left(M_{1}\right)$. The results are plotted for the same stellar model $\left(\log T_{\text {eff }}=4.2693, \log g=3.914\right)$, but with different values of $v_{\mathrm{e}}$.

azimuthal order $m$. In most of them the modes are grouped in separated regions with different $m$ values. As an example the diagrams for the pairs $\left(M_{2}, M_{1}\right)$ and $\left(F_{\lambda_{0}}, F_{\lambda_{1}}\right)$ are shown in Fig. 1.

It turned out that the diagram of $\left(M_{2}, M_{1}\right)$ is the best to assign the azimuthal order, because it is entirely independent of aspect; for any values of $i$ we have always five well separated domains with different $m$-values. The usefulness of the second moment for identification of this quantum number was already pointed out by Aerts et al. (1992). Similarly from the diagram of $\left(M_{3}, M_{1}\right)$ we can assign the azimuthal order without knowing the inclination angle, although we should be careful at small values of $i$. The diagram of $\left(F_{\lambda_{0}}, F_{\lambda_{1}}\right)$ gives poor accuracy. We studied also the effect of the amplitude of the radius variations. The spectroscopic diagrams are less sensitive to this parameter and most of them keep their diagnostic values. This result is very important especially for the multiperiodic stars, which are studied by us in another paper (Daszyńska \& Cugier, 2001). Concerning the influence of the stellar equatorial rotation it turned out that the lack of the $v_{\mathrm{e}}$-value results in the largest ambiguity. Figure 2 illustrates the effect of this parameter on the diagram employing the first and the second moment of the line profile. In two panels we have different values of the equatorial rotation. As we can see the larger value of $v_{\mathrm{e}}$ the best is the separation of regions with different azimuthal orders. The same is valid for the diagram of $\left(M_{3}, M_{1}\right)$. This method works for $v_{\mathrm{e}}>20 \mathrm{~km} / \mathrm{s}$.

In the method presented the identification of the harmonic degree $\ell$ is not unambiguous, but some other investigated diagrams are also suitable for this aim, e.g. the diagram for $\left(F W H M, V_{\mathrm{hm}}\right)$. Moreover, in some diagrams, i.e. $\left(F_{\min }, V_{\min }\right),\left(F W H M, V_{\mathrm{hm}}\right),\left(F_{\lambda_{0}}, V_{\min }\right),\left(F_{\lambda_{0}}, F_{\lambda_{1}}\right)$, the zonal modes are located in different regions with respect to the harmonic degree $\ell$, but the accuracy is poor for small inclinations $\left(i \leq 20^{\circ}\right)$ and low equatorial rotation. However, 
independent of inclination and equatorial rotation, we are always able to distinguish the zonal modes from those with $m \neq 0$. Moreover, we can say whether any mode is of prograde type $(m<0)$ or retrograde one $(m>0)$, because in most of the diagrams these types have opposite phase differences.

\section{Conclusion}

We emphasize that our results are for a star of $10 M_{\odot}$, but we expect similar ones for models with other masses. We found that the spectroscopic diagrams are especially useful for determination of the azimuthal order $m$ (the value as well as the sign), so it is complementary to the method based on the photometric diagrams. Moreover, it has very limited sensitivity to the amplitude of the radius variations, which is very promising for mode identification in the case of multiperiodic variables. The method requires high-quality spectroscopy.

\section{References}

Aerts, C., De Pauw, M., \& Waelkens, C. 1992, A\&A, 266, 294

Balona, L.A. 1986a, MNRAS, 219, 111

Balona, L.A. 1986b, MNRAS, 220, 647

Cugier, H. \& Daszyńska, J. 2001, A\&A, 377, 113

Cugier, H., Dziembowski, W.A., \& Pamyatnykh, A.A. 1994, A\&A, 266, 294

Daszyńska, J. \& Cugier, H. 2001, Odessa Astron. Publ., in press

Dziembowski, W.A. \& Pamyatnykh, A.A. 1993, MNRAS, 265, 588

Kurucz, R.L. 1996, CD-ROM No.19

Ledoux, P. 1951, ApJ, 114, 373

Watson, R.D. 1988, Ap\&SS, 140, 255,

\section{Discussion}

C. Aerts : You have to look at both amplitudes of $M_{2}$ in order to identify the modes (see Aerts et al. 1992). Depending on $m$, these amplitudes differ in importance.

J. Daszyńska : We fitted the curve consisting of six sines. Of course, amplitudes and phases of harmonic frequencies may contain important information; not only in $M_{2}$, but also in another line profile parameters. For example in the case of zonal modes, the first harmonic has the largest amplitudes except of $M_{2}$ also in $F_{\min }, F W H M, F_{\lambda_{0}}$. These diagrams are for the primary period.

C. Aerts : We have already applied many times the method of moments to identify modes in $\beta$ Cep stars from LPVs: see Aerts \& collaborators on ADS.

J. Daszyńska : Yes, I know these papers, but this is another approach: we present a new method for mode identification from LPVs. 